\title{
$\sqrt{12}$ \\ PR I F YS G OL \\ B A N G OR \\ U N IVERS IT Y
}

\section{Tuning the optical properties of luminescent down shifting layers based on organic dyes to increase the efficiency and lifetime of P3HT: PCBM photovoltaic devices}

Fernandes, Ricardo Vignoto; Urbano, Alexandre; Duarte, Jose Leonil; Bristow, Noel; Kettle, Jeffrey; Laureto, Edson

\section{Journal of Luminescence}

DOI:

10.1016/j.jlumin.2018.06.053

Published: 01/11/2018

Peer reviewed version

Cyswllt i'r cyhoeddiad / Link to publication

Dyfyniad o'r fersiwn a gyhoeddwyd / Citation for published version (APA):

Fernandes, R. V., Urbano, A., Duarte, J. L., Bristow, N., Kettle, J., \& Laureto, E. (2018). Tuning the optical properties of luminescent down shifting layers based on organic dyes to increase the efficiency and lifetime of P3HT: PCBM photovoltaic devices. Journal of Luminescence, 203, 165171. https://doi.org/10.1016/j.jlumin.2018.06.053

Hawliau Cyffredinol / General rights

Copyright and moral rights for the publications made accessible in the public portal are retained by the authors and/or other copyright owners and it is a condition of accessing publications that users recognise and abide by the legal requirements associated with these rights.

- Users may download and print one copy of any publication from the public portal for the purpose of private study or research.

- You may not further distribute the material or use it for any profit-making activity or commercial gain

- You may freely distribute the URL identifying the publication in the public portal ?

Take down policy

If you believe that this document breaches copyright please contact us providing details, and we will remove access to the work immediately and investigate your claim. 


\section{Tuning the optical properties of luminescent down shifting layers based on organic dyes to increase the efficiency and lifetime of P3HT: PCBM photovoltaic devices}

Ricardo Vignoto Fernandes ${ }^{1}$, Alexandre Urbano ${ }^{1}$, José Leonil Duarte ${ }^{1}$, Noel Bristow $^{2}$, Jeff Kettle ${ }^{2}$, Edson Laureto ${ }^{1^{*}}$

${ }^{1}$ Departamento de Física, Centro de Ciências Exatas, Universidade Estadual de Londrina, 86051-990 Londrina, PR, Brazil

2 School of Electronic Engineering, Bangor University, Dean St., Bangor, Gwynedd, LL57 1UT, Wales, UK

* contact author: laureto@uel.br

Abstract

Mixtures of luminescence downshifting (LDS) materials has been used to increase the efficiency of poly(3-hexylthiophene-2,5-diyl):phenyl-C61-butyric acid methyl ester (P3HT:PC61BM) bulk heterojunction solar cell. This layers convert more energetic photons to lower energies that are better matched with wavelength peak of the external quantum efficiency (EQE) of a $\mathrm{P} 3 \mathrm{HT}: \mathrm{PC}_{61} \mathrm{BM}$ solar cell. Experimental studies were used to optimise the optical properties of LDS layers including the maximum of absorbance and the photoluminescence quantum yield (PLQY). To provide the significant improvements, combinations of LDS mixtures were prepared to provide the greatest absorption and PLQY. The approach is shown to simultaneously improve the photocurrent and increase the lifetime of the device by absorbing UV light. By optimising the optical properties of the LDS mixture, a relative increase of about $20 \%$ in the photocurrent density produced by the P3HT:PCBM cell could be achieved, which to our knowledge is one of the most significant reported for OPVs.

Keywords: Organic photovoltaics. Organic dyes. Photoluminescence quantum yield. External quantum efficiency. Luminescence downshifting. 


\section{Introduction}

Due to the increasing global energy demand [1, 2], the development of alternative sources of energy has become an increasingly urgent matter. Given the environmental problems related to the use of fossil fuels in energy generation [3, 4], it is recommended that these alternative sources are renewable, sustainable and environmentally friendly. Energy generation through the photovoltaic effect meets these requirements and is already well established [5] with further photovoltaic (PV) technology developments emerging in order to reduce production costs further and enhancing their accessibility [6]. Among them, solar cells based on organic materials are currently the subject of intense research and development $[7,8]$. A representative device is one whose active layer consists of the conjugated polymer poly-3 hexyl-thiophene (P3HT) and the fullerene phenylC61-butyric acid methyl ester (PCBM) forming a bulk heterojunction [9-11]. These devices are based on materials in solution that have a broad spectrum of optical absorption, good chemical stability, and can be processed by printing techniques on flexible substrates $[12,13]$, which makes them relatively versatile products that can be used in applications that are unattainable to conventional siliconbased solar cells [14]. However, there are still major challenges to make organic photovoltaic technology (OPV) commercially attractive, the most important being issues related to efficiency and lifetime.

An elegant strategy to improve efficiency and lifetime is by utilising luminescence downshifting (LDS) effect [15-18]. This approach employs a layer containing a luminescent material that absorbs photons at higher energies, where current generation is low, and re-emits at lower energy photons where the external quantum efficiency (EQE) of the OPV is at its highest. For OPVs, the proof of concept for LDS application has already been established by Kettle et al. [19]. The LDS layer was shown to increase the lifetime of the OPV by reducing UV -induced degradation, and showed performance better than commercial UV filters owing to reduced reflection. Whilst the approach showed significantly enhanced lifetime over a solar cell without a UV filter, only a slight increment in the photocurrent was possible over devices with no UV filters. The optimisation of luminescent material selection has already been shown to lead to significant improvements in current density of PV technologies such as those based on c-Si 
$[20,21]$, CdTe $[22,23]$ and CIGS [24] thin films, and in DSSCs $[25,26]$ and further optimisation could lead to greater enhancement OPV devices.

Modelling of the effect of luminescent materials has been conducted by number of authors including one proposed by Rothemund [27]. The model considered a number of optical losses including reflection, re-absorption, and front and lateral side emission; and modelled a number of figures of merit including absorbance, PLQY and emission spectral matching (ESM), which measures the overlap of the re-emitted photon to the EQE of the solar cell [28].

One class of LDS materials that are showing increasing promise are based upon organic dyes. They possess a high absorptivity and PLQY, are abundant and their cost is lower than other candidates, such as Europium complexes, quantum dots or nanophosphors [29]. However, as pointed out by Uekert et al. [30], organic dyes generally have narrow absorption bands and, as a rule of thumb in organic compounds, exhibit relatively low photo stability, mainly with exposure to UV radiation. The purpose of this work is to optimise the properties of LDS layers based on organic dyes to produce a significant further increase in the current density generated by photovoltaic cells having P3HT:PCBM as the active layer. Simultaneously, it is intended that the LDS layer should also extend the lifetime of the photovoltaic cell. For this, the Rothemund model has been used to select suitable materials to act as LDS layers, and experimental work was developed to maximize the optical properties of these layers in order to promote an improvement in the photocurrent produced by the cell.

\section{Experimental}

The materials used in the work were Coumarim 153 (C153), Coumarim 7 (C7), Kremer Blue (KB), $\mathrm{AlQ}_{3}$ and Europium Complex (Eu), as shown in Table 1. Figure 1 shows the chemical structure of these compounds. These materials were chosen because their absorbance spectra were below $450 \mathrm{~nm}$ (thus filtering UV light) and the photoluminescence spectrum was within the range of best EQE response of a solar cell based on P3HT:PCBM. Kremer Blue luminescent material was purchased from Kremer Pigmente ${ }^{\circledR}$. All other materials were supplied by Sigma Aldrich $\circledast$ and were used as received. 
Table 1: Luminescent materials selected for this work.

\begin{tabular}{|l|l|l|l|l|}
\hline Code & Dye & $\begin{array}{l}\text { Supplier } \\
\text { company }\end{array}$ & $\begin{array}{l}\text { Molecular } \\
\text { Weigth }(\mathrm{g} / \mathrm{mol})\end{array}$ & $\begin{array}{l}\text { Chemical } \\
\text { Family }\end{array}$ \\
\hline C153 & Coumarin 153 & Sigma Aldrich & 309.28 & Coumarin dye \\
\hline C7 & Coumarin 7 & Sigma Aldrich & 333.38 & Coumarin dye \\
\hline KB & Kremer Blue & $\begin{array}{l}\text { Kremer } \\
\text { Pigmente }\end{array}$ & n/a & $\begin{array}{l}\text { Naphthalimide } \\
\text { dye }\end{array}$ \\
\hline Alq3 & Alq3 & Sigma Aldrich & 459.43 & $\begin{array}{l}\text { Metal/organic } \\
\text { complex }\end{array}$ \\
\hline Eu & $\begin{array}{l}\text { Europium } \\
\text { complex }\end{array}$ & Sigma Aldrich & 1001.91 & $\begin{array}{l}\text { Lanthanide } \\
\text { complex }\end{array}$ \\
\hline
\end{tabular}<smiles>O=c1cc(C(F)(F)F)c2cc3c4c(c2oc1=O)CCCN4CCC3</smiles>

Coumarin 153

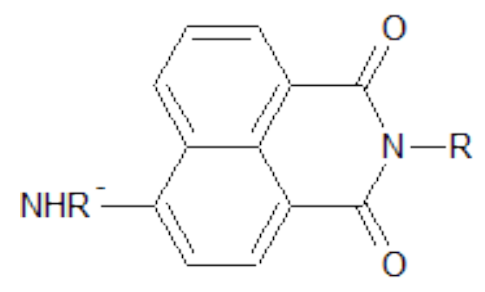

Napthalimide Dyes

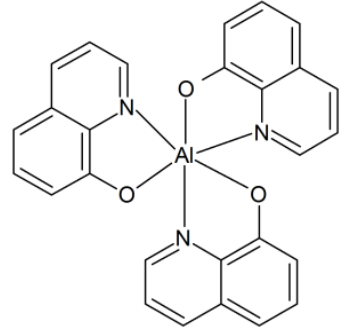

$\mathrm{Alq}_{3}$

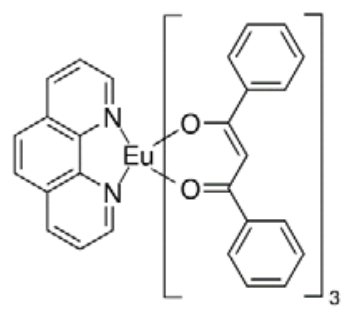

Europium complex 
Figure 1: Chemical structures of the materials listed in Table 1.

LDS solutions for coating were prepared by mixing the luminescent materials with commercially available poly (methyl methacrylate) (PMMA) dissolved in chlorobenzene supplied by Sigma Aldrich, at a concentration of $1 \%$ by weight (other concentrations were used during tests aimed to improve the optical properties of the LDS layers, as presented in the Supplemental Information file - SI). Several concentrations of the solutions were used, as will be seen in the next section. The blended solutions were left under magnetic stirring on a hot plate at $50{ }^{\circ} \mathrm{C}$ overnight. To undertake the optical characterisation, the substrates were quartz laminas, as light emission occurs above $300 \mathrm{~nm}$. Substrate cleaning was conducted by immersing the lamina in chloroform for 10 minutes in an ultrasonic cleaner and drying with compressed air flow. The films were produced by drop casting, using $50 \mu \mathrm{L}$ of the solution, followed by a $60{ }^{\circ} \mathrm{C}$ heat treatment for 30 minutes to evaporate solvent residues.

For absorbance measurements, a DT-Mini-2-GS source and the USB2000+ mini-spectrometer, both from Ocean Optics ${ }^{\circledR}$ were used. The same spectrometer was also used for photoluminescence measurements, where the excitation beam was a Coherent ${ }^{\circledR}$ laser with emission at $375 \mathrm{~nm}$. For PLQY measurements, a Coherent ${ }^{\circledR}$ laser with emission at $375 \mathrm{~nm}$, an integrating sphere from Labsphere ${ }^{\circledR}$ and a USB2000+ mini-spectrometer from Ocean Optics ${ }^{\circledR}$ were used. For the stability tests, the films were exposed to sunlight during daylight hours (from 10 am to 2 pm) in Londrina, Brazil (2319'36" S, $\left.51^{\circ} 12^{\prime} 10^{\prime \prime} \mathrm{W}\right)$, in which the solar radiation intensity was $(8.7 \pm 0.5) 10^{4}$ lux. During the tests, the temperature and relative air humidity was around $30{ }^{\circ} \mathrm{C}$ and $75 \%$, respectively, and remained approximately constant during test. The procedure is according the ISOS-O-2 outdoor test protocol [31].

The photovoltaic devices were prepared on indium-tin oxide (ITO) coated glass substrates with a surface resistivity of $20 \Omega / \mathrm{cm}^{2}$. The substrates were cleaned using deionized water, acetone, and isopropyl alcohol (IPA), 5 minutes each in an ultrasonic cleaner and then treated in a UV-ozone reactor at ambient atmosphere for 15 minutes. The poly (3,4-ethylenedioxythiophene):poly (styrene 
sulfonate) (PEDOT:PSS) conductive layer was deposited by spin coating at 4500 $\mathrm{rpm}$ for $30 \mathrm{~s}$, followed by a heat treatment at $150{ }^{\circ} \mathrm{C}$ for 60 minutes to remove the residual solvent. The solution of the $\mathrm{P} 3 \mathrm{HT}: \mathrm{PC}_{61} \mathrm{BM}$, in the ratio of $17: 13$ by mass, was dissolved in chlorobenzene at a concentration of $30 \mathrm{mg} / \mathrm{ml}$. The solution was stirred for $24 \mathrm{~h}$ on the hotplate at $60^{\circ} \mathrm{C}$. The active layer was deposited by spin coating at $1500 \mathrm{rpm}$ for $60 \mathrm{~s}$. Subsequently, a heat treatment was carried out at $150^{\circ} \mathrm{C}$ for 30 minutes in ambient atmosphere. The electrode was a $150 \mathrm{~nm}$ film of aluminum (Al) deposited by thermal evaporation at pressure around $10^{-5} \mathrm{mbar}$. The area of the devices was $0.4 \mathrm{~cm}^{2}$.

The devices were tested using a low-cost solar simulator from $A B E T$ Technologies ${ }^{\circledR}$ with $100 \mathrm{~mW} / \mathrm{cm}^{2}$ light intensity, according to the AM1.5G standard. In order to ensure consistency of results, only devices with a standard deviation within $10 \%$ of the mean value were used in the experiment. The electrical characterization of the devices was made before and after deposition of the LDS layer, in order to evaluate the difference in the photo-generated electric current density. It is worth noting that the LDS layer was applied always onto the outside of the glass substrate, i.e. the outer side of the device where the light is incident.

\section{Results and Discussion}

To be effective as a LDS layer, the luminescent material must possess absorption outside the EQE of the PV. Furthermore, photon re-emission should be within the region of higher EQE of the device. Based upon these criteria, five commercial organic dyes were selected for the development of this work, whose absorbance and PL spectra are shown in Figure 2 (a) and (b), respectively. In this figure, the EQE as a function of wavelength for the solar cell used in this work is overlaid. It can be seen from the figure that all dyes possess the prerequisites required for a luminescent material to be employed as LDS layer in the P3HT:PCBM OPV, as the absorption peaks are all below $450 \mathrm{~nm}$ and re-emission mostly occurs at wavelengths greater than $450 \mathrm{~nm}$. 

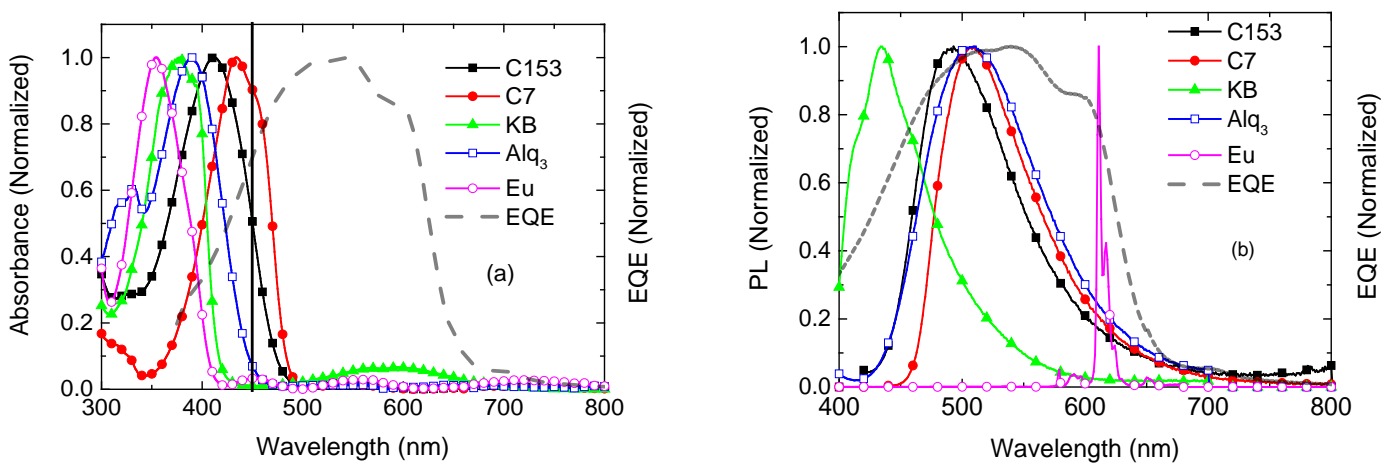

Figure 2: (a) Absorbance spectra of the LDS materials selected for this work (continuous lines) and the EQE curve of the device (dashed line). (b) Photoluminescence spectra of the LDS materials (continuous lines) and EQE the device (dashed line).

It is possible to estimate the efficiency of the LDS layer on a photovoltaic device using the optical model proposed by Rothemund [27], which is based on the following equation:

$$
E Q E_{L D S}(\lambda)=E Q E_{\text {ref }}(\lambda) * T+n_{\text {loss }} *(1-T) * E S M * P L Q Y
$$

with $T=10^{-A(\lambda)}$ and $\mathrm{A}(\lambda)$ being the absorbance of the LDS layer. Given the PLQY value, and calculating ESM using the PL spectrum of the luminescent layer and the EQE curve of the device $\left(\operatorname{EQE}_{r e f}(\lambda)\right)[28]$, we can estimate the EQE curve of the device after the inclusion of the LDS layer, represented by $\operatorname{EQE} \operatorname{LDS}(\lambda)$, provided that the value of the nloss parameter is known. This parameter takes into account all optical losses generated by the inclusion of the LDS layer on the device, related to the reflection, front and lateral side escape, and re-absorption. In order to estimate the value of nloss, the EQEref and EQELDS curves of P3HT:PCBM devices were used (measured in earlier work), before and after the inclusion of a LDS layer, respectively [31]. Thus, the experimental curve $\operatorname{EQE} \operatorname{LDS}(\lambda)$ was adjusted by the curve generated by eq. (1), using nloss as the fitting parameter. The details of this procedure are contained in the SI file. The best fittings were obtained for nloss having values between 0.5 and 0.7 . 
Assuming that the same values of nloss can be used for the LDS layers developed in the present work, the model was then applied to simulate the EQE curves taking into account the optical properties of the luminescent material, as well as the EQE curve of the P3HT:PCBM. From these simulations, it was observed that there is a compromise between the absorbance of the layer and its PLQY value. For example, a high absorbance can lead to a "filtering effect", which leads to a decrease in the EQE. On the other hand, a low PLQY value does not produce a sufficient amount of downshifted photons to generate a sizeable increase in photocurrent generation. Based upon data of this model, an experimental optimisation procedure was undertaken in order to maximize the PLQY of the luminescent layer, whilst maintaining the maximum absorbance close to its best value obtained from the simulations, which was between 0.7 and 1.2. The best PLQY values for LDS layers of the luminescent materials produced in this work are shown in Table 2, along with the parameters used for the production of the layer.

Table 2: Optimised values for PLQY of the LDS layers composed by the luminescent materials. The estimated error in the PLQY determination is $10 \%$.

\begin{tabular}{|c|c|c|c|}
\hline Material & $\begin{array}{c}\text { Concentration } \\
(\mathrm{mg} / \mathrm{mL}) \text { in PMMA 1\% }\end{array}$ & $\begin{array}{c}\text { Dripped volume } \\
(\mu \mathrm{L})\end{array}$ & PLQY (\%) \\
\hline C153 & 0.5 & 30 & 37.4 \\
\hline C7 & 0.5 & 30 & 20.2 \\
\hline KB & 0.5 & 40 & 30.0 \\
\hline Alq3 & 1.0 & 100 & 55.5 \\
\hline Eu & 1.0 & 40 & \\
\hline
\end{tabular}

The secondary purpose of the LDS layer is to improve the stability of the OPV device as it acts as a UV filter, limiting photo-degradation [19, 26, 32]. However, the photo stability of the LDS layer should be also evaluated as this 
can change with light exposure. Therefore, the LDS layers were exposed to outdoor sunlight using the ISOS-O-2 outdoor test protocol (which is detailed in the Experimental Section). Absorbance curves were measured before and after exposure, and the results are shown in Figure 3.
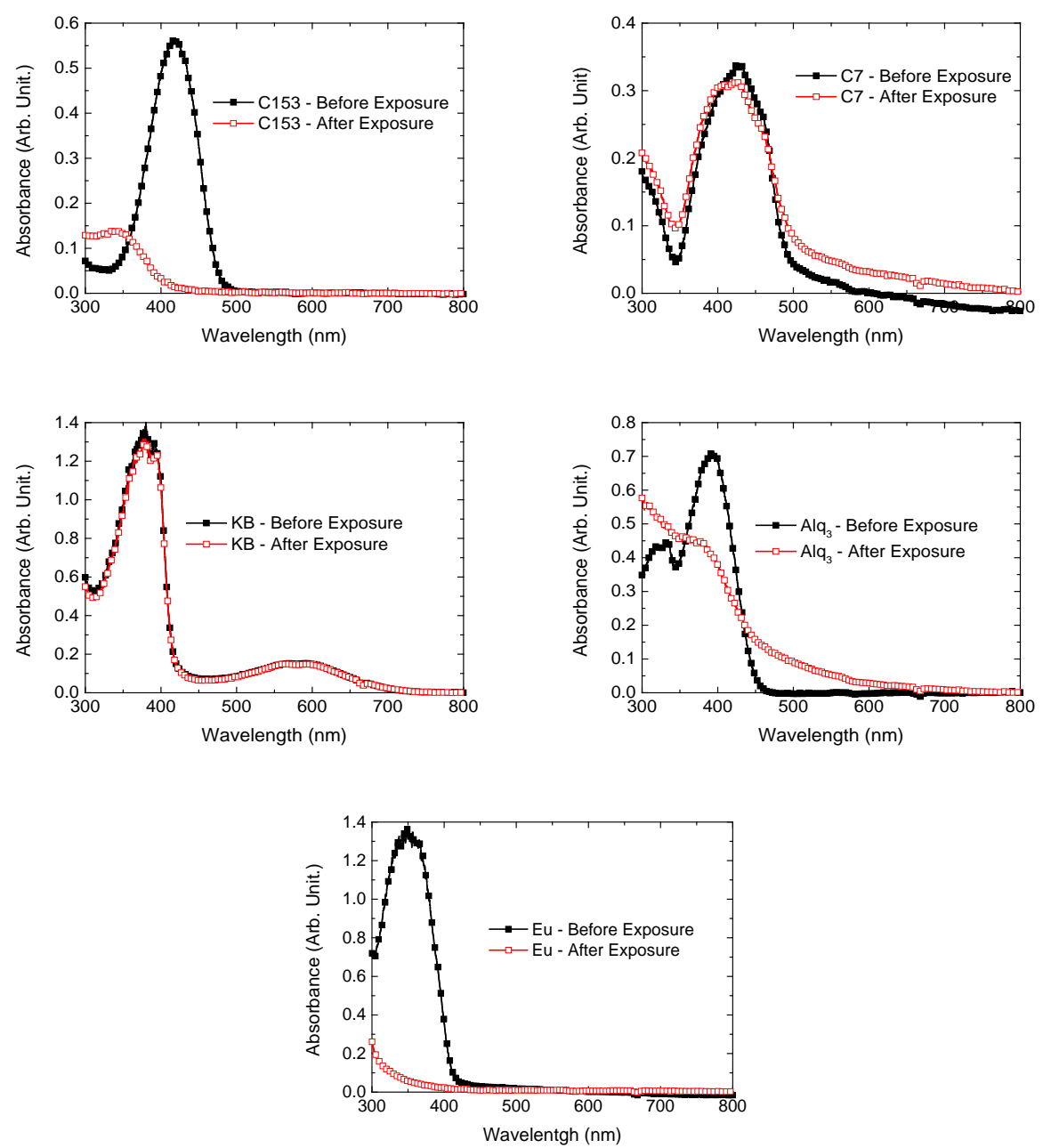

Figure 3: Comparison between absorbance spectra of the samples listed in table 2 , before and after the solar exposure (conditions were indicated in the experimental section).

From the data in Figure 3, it was observed that the KB material demonstrated no significant change in its absorbance after exposure to solar radiation, however, the layer composed of the $\mathrm{KB}$ material also possesses the lowest PLQY value (see table 2). The C153 and Eu compounds, which had higher emission efficiencies, were the ones that suffered the highest decreases in optical absorbance during the outdoor testing period. 
The low photostability of the Eu complex is in agreement with the literature, since it is one of the main problems related to the lanthanide complexes $[33,34]$. The photodegradation of compounds similar to C153 was already traced, and occurs at the bonding between the carbon and nitrogen atoms (see Figure 1), through a double bonding with an oxygen atom [35]. This type of bonding does not occur in C7-type compounds. On the other hand, the high stability of KB compound may be related to the more compact form of the molecule. As discussed by Manceau et al., compounds with more compact structures are less susceptible to the photodegradation [36].

In order to obtain a compromise between higher efficiency and greater robustness against photo-degradation effects, LDS layers were produced from mixtures of luminescent materials defined in table 2. Again, an experimental optimisation routine was carried out by varying the production and deposition parameters for the mixed LDS layers with the goal to maximize the PLQY while maintaining the best values of absorbance of the layer (see SI file for further details). The results of this optimisation trial are presented in Table 3.

Table 3: Parameters for the production of LDS layers composed by mixtures of the luminescent materials, and their measured PLQY values. The estimated error in the PLQY measurement is $\pm 10 \%$.

\begin{tabular}{|c|c|c|c|}
\hline Mixtures & $\begin{array}{c}\text { Concentration } \\
(\mathrm{mg}: \mathrm{mg} / \mathrm{mL}) \text { in PMMA 1\% }\end{array}$ & $\begin{array}{c}\text { Dripped } \\
\text { Volume }(\mu \mathrm{L})\end{array}$ & PLQY (\%) \\
\hline KB:C7 & $0.17: 0.17$ & 50 & 38.8 \\
\hline KB:Eu & $0.17: 0.33$ & 50 & 45.2 \\
\hline KB:C153 & $0.17: 0.17$ & 50 & 88.0 \\
\hline
\end{tabular}

It is notable that the PLQY values using the $\mathrm{KB}: \mathrm{C} 153$ and $\mathrm{KB}: \mathrm{C} 7$ mixtures are substantially higher than those presented by their individual components shown in table 2. The PLQY of the KB:Eu mixture presented a lower value than 
the Eu layer (when comparing the values in Tables 2 and 3). The changes in PLQY value can be related to two main factors: (i) firstly, the concentration of LDS material, and layers produced from more dilute solutions tend to present higher PLQY values. This may be mainly correlated to a decrease in the reabsorption effects; (ii) secondly, energy transfer processes [37, 38] which may lead to an increase in the PLQY value of the mixtures. In Figure S2 (SI file) the absorbance and PL curves for both the individual components and their mixtures are shown for the compounds listed in table 3 . It can be seen that there is a high degree of overlap between the KB luminescence with the absorbance of C153 and $\mathrm{C7}$, which is one of the prerequisites for the energy transfer process [38] between the donor (KB) and the acceptor (C153 or $\mathrm{C} 7$ ). However, this overlap does not occur in the KB:Eu mixture, which indicates substantially reduced energy transfer between the compounds in this case. By combining the energy transfer efficiency with the relative composition in the mixtures, it is possible that higher PLQY and more extended absorbance bands can be achieved, producing even better results for mixtures of organic dyes as LDS layers.

In order to evaluate the robustness of the LDS layers composed by mixture of luminescent materials, the layers were also exposed to solar radiation using the same protocol stated above. The absorbance curves of the mixed materials LDS layers, before and after solar exposure, are shown in Figure 4.
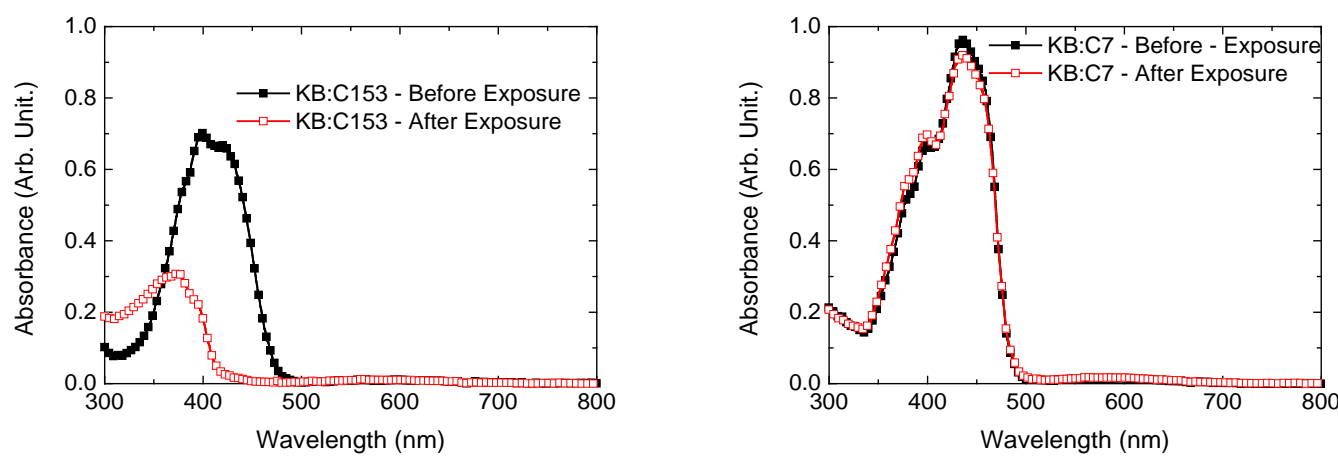


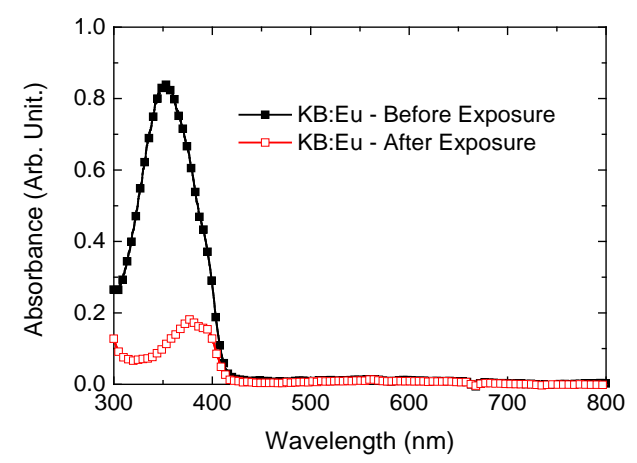

Figure 4: Comparison between absorbance spectra of the LDS layers composed by mixture of the luminescent materials, before and after the solar exposure (conditions were indicated in the experimental section).

As expected, the mixture that presented the greatest robustness was $K B: C 7$, since these two materials individually had already been shown to be the most resistant to photo-induced degradation. On the other hand, the KB:Eu mixture was the most affected by irradiation and showed the biggest decrease in absorption. However, the KB:C153 mixture, which has the highest PLQY, remained at approximately $50 \%$ of its initial maximum absorbance after the irradiation period. It is noteworthy that the LDS layers were exposed to irradiation, oxygen and water vapour without any further UV protection or environmental encapsulation. Commercial OPVs need an encapsulation to protect them against the deleterious environmental effects [39, 40]. Thus, the inclusion of the LDS layer can be performed on the device prior to its encapsulation, so that the LDS layer itself could be protected from the degrading effects of the environment in a commercial device.

Even though the KB:C153 mixture does not have the greatest stability, it may be that, due to its high PLQY value, this LDS layer could result in a significant gain in the current density in a P3HT:PCBM OPV. In order to evaluate the LDS properties of the KB:C153 mixture, the EQE curve of the device was simulated, according to eq. (1), by considering the optical gain achieved using this LDS mixture. For the purpose of comparison, a simulation was also made using the KB material as the luminescent layer, whose parameters were shown in table 2. The results of the simulations are shown in Figure 5. 

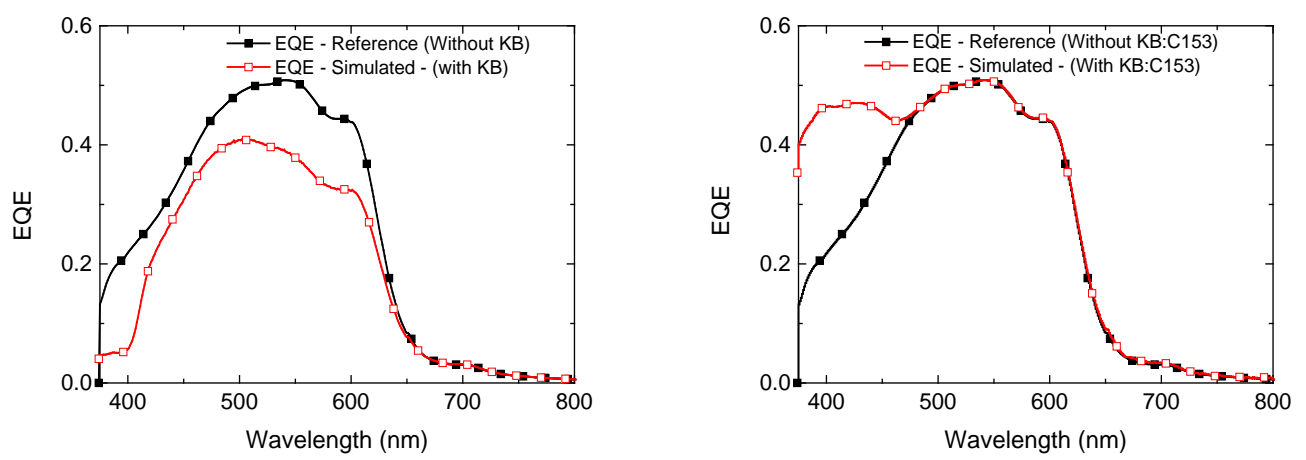

Figure 5: Simulated EQE curve (red line) of P3HT:PCBM based photovoltaic devices with (a) KB and (b) KB:C153 LDS layer. The EQE curve of the device without LDS layer is shown for comparison (black line).

The simulations demonstrate that the inclusion of a LDS layer containing the KB:C153 mixture leads to a significant improvement of the EQE at wavelengths shorter than $480 \mathrm{~nm}$. By using the KB material on its own, a marked drop in the EQE of the OPV is observed as result of the low PLQY of this material. By taking the integral of the EQE curve in the wavelength range is equivalent to the current density photo-generated by the device, it can be predicted that a higher current density will be obtained by applying an LDS layer of the KB:C153 mixture on a P3HT:PCBM-based photovoltaic device.

In order to verify the effect of the LDS layer experimentally, P3HT:PCBM-based solar cells were manufactured and LDS layers of both the $\mathrm{KB}: \mathrm{C} 153$ mixture and the KB material were applied onto such devices. The characteristic curves of these devices were then obtained, before and after the inclusion of the LDS layers, and are shown in Figure 6. 

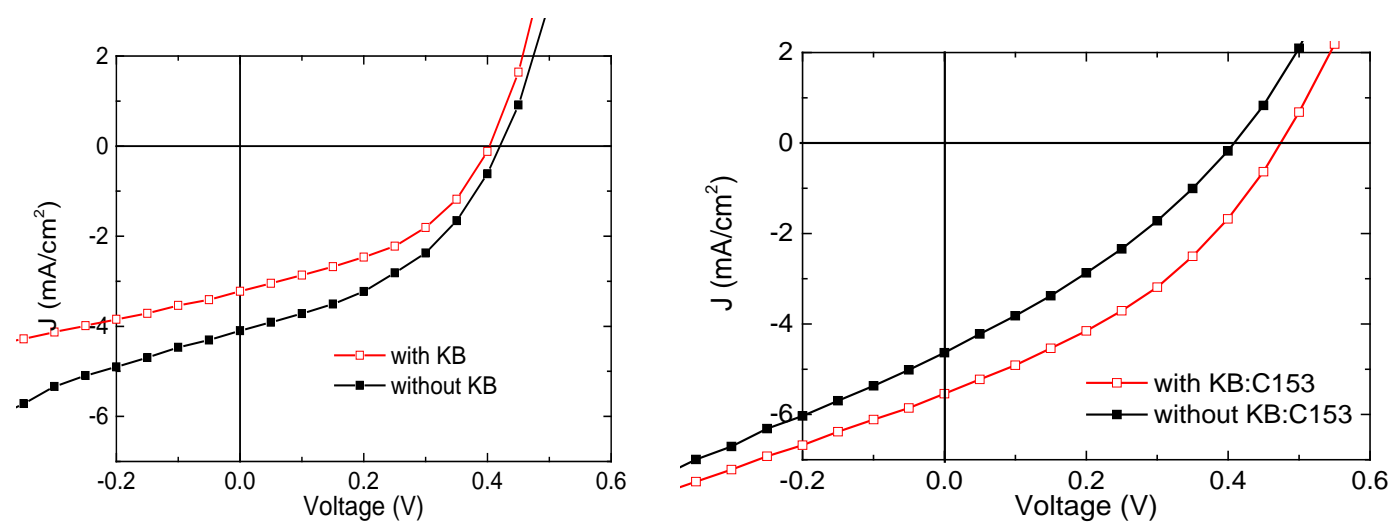

Figure 6: Electrical characterization of the OPVs before and after the inclusion of the LDS layer, (left) KB and (right) KB:C153 mixture. The results are an average for twelve samples.

It can be clearly seen that the photocurrent generation decreased by using the $\mathrm{KB}$ layer, but increased with the KB:C153 mixture. Table 4 compares the values of the relative variation of the short-circuit current density (Jsc) for the devices due to the inclusion of the LDS layers. There is a very good agreement between the variations obtained by the integration of the simulated EQE curve with the values obtained experimentally, showing that the model used in this work is effective for the evaluation of the effect of LDS layers on the efficiency of photovoltaic devices.

Table 4: Comparison between simulated and experimental results for the relative variation of the current density photogenerated by the devices after inclusion of the LDS layers.

\begin{tabular}{|c|c|}
\hline LDS layer & $\begin{array}{c}\text { (Jsc (With LDS) - Jsc (Without LDS))/ Jsc } \\
\text { (Without LDS) }\end{array}$ \\
\hline KB - Simulated & $(\%)$ \\
\hline KB - Experimental & -21.7 \\
\hline KB:C153 - Simulated & $-21 \pm 2$ \\
\hline
\end{tabular}


In the case of the $\mathrm{KB}: \mathrm{C} 153$ mixture, the relative increase in Jsc was about $20 \%$, which is significantly greater than the previously reported for LDS layers applied to OPV's [19, 26, 32]. Such an improvement in the current density shows optimisation the optical properties of the luminescent material (or mixtures of these materials) is imperative to design efficient LDS layers.

\section{Conclusion}

Organic dyes have been used as LDS layers for OPV devices based on a P3HT:PCBM active layer. After experimental optimisation work, the LDS layer achieved a $20 \%$ increase in the photo-generated current density, which represents a substantial change in comparison to the previously reported results of using an LDS layer in OPV devices. The increase of the current density was compared with simulations of the EQE curve of the devices taking into account the inclusion of the LDS layer. The model proved to be effective as a strategy to predict the effect of the LDS layer onto the EQE of the device. It is also possible to combine the LDS properties with the function of protecting the device against the UV degradation. Therefore outdoor measurements were conducted to evaluate stability and the optimum stability of the LDS layer was identified based upon these organic dyes.

\section{Acknowledgments}

The authors would like to thank CNPq and CAPES for financial support. JK and NB would like to acknowledge the European Regional Development Fund (ERDF) and the Welsh European Funding Office (WEFO) for funding the 2nd Solar Photovoltaic Academic Research Consortium (SPARC II). 


\section{References}

1. Holmberg, K., et al., Global energy consumption due to friction and wear in the mining industry. Tribology International, 2017. 115: p. 116-139.

2. Pablo-Romero, M.d.P., R. Pozo-Barajas, and R. Yñiguez, Global changes in residential energy consumption. Energy Policy, 2017. 101: p. 342-352.

3. Lazkano, I., L. Nøstbakken, and M. Pelli, From fossil fuels to renewables: The role of electricity storage. European Economic Review, 2017. 99: p. 113-129.

4. Meehan, G., Thank You Fossil Fuels and Good Night: The 21st Century's Energy Transition. 2017: University of Utah Press.

5. Zhao, Z.-Y., H.-J. Yang, and J. Zuo, Evolution of international trade for photovoltaic cells: A spatial structure study. Energy, 2017. 124: p. 435446.

6. Rauf, I.A. and P. Rezai, A review of materials selection for optimized efficiency in quantum dot sensitized solar cells: A simplified approach to reviewing literature data. Renewable and Sustainable Energy Reviews, 2017. 73: p. 408-422.

7. Jung, J.W., et al., Recent progress in high efficiency polymer solar cells by rational design and energy level tuning of low bandgap copolymers with various electron-withdrawing units. Organic Electronics, 2016. 31: p. 149170.

8. Wang, Q., et al., Progress in emerging solution-processed thin film solar cells-part I: polymer solar cells. Renewable and Sustainable Energy Reviews, 2016. 56: p. 347-361.

9. Ameri, T., et al., Organic tandem solar cells: a review. Energy \& Environmental Science, 2009. 2(4): p. 347-363.

10. Dang, M.T., L. Hirsch, and G. Wantz, P3HT: PCBM, best seller in polymer photovoltaic research. Advanced Materials, 2011. 23(31): p. 3597-3602.

11. Obuchovsky, S., et al., Morphology visualization of P3HT: Fullerene blends by using subsurface atomic layer deposition. Organic Electronics, 2017. 49: p. 234-241. 
12. Seo, J.H., et al., Cold Isostatic-Pressured Silver Nanowire Electrodes for Flexible Organic Solar Cells via Room-Temperature Processes. Advanced Materials, 2017. 29(30).

13. Etxebarria, I., J. Ajuria, and R. Pacios, Solution-processable polymeric solar cells: A review on materials, strategies and cell architectures to overcome 10\%. Organic Electronics, 2015. 19: p. 34-60.

14. Green, M.A., Third generation photovoltaics. 2006: Springer.

15. Talewar, R., C. Joshi, and S. Moharil, Enhancing the photovoltaic performance of $\mathrm{CdTe} / \mathrm{CdS}$ solar cell via luminescent downshifting using K2SiF6: Mn4+ phosphors. AIP Conference Proceedings, 2016. 1731(1): p. 140019.

16. Richards, B., Enhancing the performance of silicon solar cells via the application of passive luminescence conversion layers. Solar energy materials and solar cells, 2006. 90(15): p. 2329-2337.

17. Klampaftis, E., et al., Enhancing the performance of solar cells via luminescent down-shifting of the incident spectrum: A review. Solar Energy Materials and Solar Cells, 2009. 93(8): p. 1182-1194.

18. Anizelli, H.S., et al., Application of luminescence downshifting materials for enhanced stability of $\mathrm{CH} 3 \mathrm{NH} 3 \mathrm{Pbl} 3(1-\mathrm{x}) \mathrm{Cl} 3 \mathrm{x}$ perovskite photovoltaic devices. Organic Electronics, 2017. 49: p. 129-134.

19. Kettle, J., et al., Printable luminescent down shifter for enhancing efficiency and stability of organic photovoltaics. Solar Energy Materials and Solar Cells, 2016. 144: p. 481-487.

20. Barman, B., et al., Study of formation and influence of surface plasmonic silver nanoparticles in efficiency enhancement for c-Si solar cells. AIP Conference Proceedings, 2016. 1731(1): p. 050142.

21. Ahmed, H., J. Doran, and S. McCormack, Increased short-circuit current density and external quantum efficiency of silicon and dye sensitised solar cells through plasmonic luminescent down-shifting layers. Solar Energy, 2016. 126: p. 146-155.

22. Kanevce, A., et al., The roles of carrier concentration and interface, bulk, and grain-boundary recombination for $25 \%$ efficient CdTe solar cells. Journal of Applied Physics, 2017. 121(21): p. 214506. 
23. Ross, D., et al., The impact of luminescent down shifting on the performance of CdTe photovoltaics: impact of the module vintage. IEEE Journal of Photovoltaics, 2014. 4(1): p. 457-464.

24. Solodovnyk, A., et al., Optimization of Solution-Processed Luminescent Down-Shifting Layers for Photovoltaics by Customizing Organic Dye Based Thick Films. Energy Technology, 2016. 4(3): p. 385 - 392.

25. Musyaro'ah, et al., Fabrication and characterization dye sensitized solar cell (DSSC) based on TiO2/SnO2 composite. AIP Conference Proceedings, 2017. 1788(1): p. 030062.

26. Bella, F., et al., Performance and stability improvements for dye-sensitized solar cells in the presence of luminescent coatings. Journal of Power Sources, 2015. 283: p. 195-203.

27. Rothemund, R., Optical modelling of the external quantum efficiency of solar cells with luminescent down-shifting layers. Solar Energy Materials and Solar Cells, 2014. 120: p. 616-621.

28. Alonso-Álvarez, D., et al., Luminescent down-shifting experiment and modelling with multiple photovoltaic technologies. Progress in Photovoltaics: Research and Applications, 2015. 23(4): p. 479-497.

29. Chen, J.-Y., et al., Efficiency improvement of Si solar cells using metalenhanced nanophosphor fluorescence. Solar Energy Materials and Solar Cells, 2014. 120: p. 168-174.

30. Uekert, T., et al., Nanostructured organosilicon luminophores in highly efficient luminescent down-shifting layers for thin film photovoltaics. Solar Energy Materials and Solar Cells, 2016. 155: p. 1-8.

31. Reese, M.O., et al., Consensus stability testing protocols for organic photovoltaic materials and devices. Solar Energy Materials and Solar Cells, 2011. 95(5): p. 1253-1267.

32. Ricardo Vignoto Fernandes, N.B., Vasil Stoichkov, Helder Scapin Anizelli, José Leonil Duarte, Edson Laureto, Jeff Kettle, Development of multidye UV filters for OPVs using luminescent materials. Journal of Physics D: Applied Physics, 2017. 50(2): p. 025103.

33. Moretti, E., et al., Concentration quenching and photostability in Eu (dbm) 3phen embedded in mesoporous silica nanoparticles. Journal of Luminescence, 2014. 146: p. 178-185. 
34. Gameiro, C., et al., Lanthanide complexes dispersed in enamel: a promising new material for photonic devices. Journal of alloys and compounds, 2001. 323: p. 820-823.

35. Natal'ya, A.K. and O.L. Kaliya, The photochemistry of coumarins. Russian Chemical Reviews, 1992. 61(7): p. 683.

36. Manceau, M., et al., Photochemical stability of $\pi$-conjugated polymers for polymer solar cells: a rule of thumb. Journal of Materials Chemistry, 2011. 21(12): p. 4132-4141.

37. Renzi, W., et al., Analysis and control of energy transfer processes and luminescence across the visible spectrum in PFO: P3OT blends. Journal of Materials Science: Materials in Electronics, 2017. 28(23): p. 1775017760.

38. Valeur, B. and M.N. Berberan-Santos, Molecular fluorescence: principles and applications. 2012: John Wiley \& Sons.

39. dos Reis Benatto, G.A., et al., Carbon: the ultimate electrode choice for widely distributed polymer solar cells. Advanced Energy Materials, 2014. 4(15).

40. Moudam, O., et al., Application of UV-absorbing silver (i) luminescent down shifter for PTB7 organic solar cells for enhanced efficiency and stability. RSC Advances, 2015. 5(16): p. 12397-12402. 


\section{TABLE CAPTIONS}

Table 1: Luminescent materials selected for this work.

Table 2: Optimised values for PLQY of the LDS layers composed by the luminescent materials. The estimated error in the PLQY determination is $10 \%$.

Table 3: Parameters for the production of LDS layers composed by mixtures of the luminescent materials, and their measured PLQY values. The estimated error in the PLQY measurement is $\pm 10 \%$.

Table 4: Comparison between simulated and experimental results for the relative variation of the current density photogenerated by the devices after inclusion of the LDS layers.

\section{FIGURE CAPTIONS}

Figure 1: Chemical structures of the luminescent materials used as LDS layers.

Figure 2: (a) Absorbance spectra of the LDS materials selected for this work (continuous lines) and the EQE curve of the device (dashed line). (b) Photoluminescence spectra of the LDS materials (continuous lines) and EQE the device (dashed line).

Figure 3: Comparison between absorbance spectra of the samples listed in table 2, before and after the solar exposure (conditions were indicated in the experimental section).

Figure 4: Comparison between absorbance spectra of the LDS layers composed by mixture of the luminescent materials, before and after the solar exposure (conditions were indicated in the experimental section).

Figure 5: Simulated EQE curve (red line) of P3HT:PCBM based photovoltaic devices with (a) KB and (b) KB:C153 LDS layer. The EQE curve of the device without LDS layer is shown for comparison (black line). 
Figure 6: Electrical characterization of the OPVs before and after the inclusion of the LDS layer, (left) KB and (right) KB:C153 mixture. The results are an average for twelve samples. 


\section{SUPPLEMENTAL INFORMATION}

I. EQE curve simulation to evaluate the nloss parameter.

The first step of this work was to reproduce the EQE curves obtained by Fernandes et al. [32], from simulations based on the eq. (1) (see main text), aiming to evaluate the nloss, a parameter that takes account the optical losses due to the LDS layer application. The simulation requires as inputs the absorbance and photoluminescence spectra of the material to be used as the LDS layer, the PLQY value and the ESM of the layer, as well as the EQE curve of the reference cell on which the LDS layer will be applied. In this work, the active layer was chosen to be based on the P3HT:PCBM bulk heterojunction.
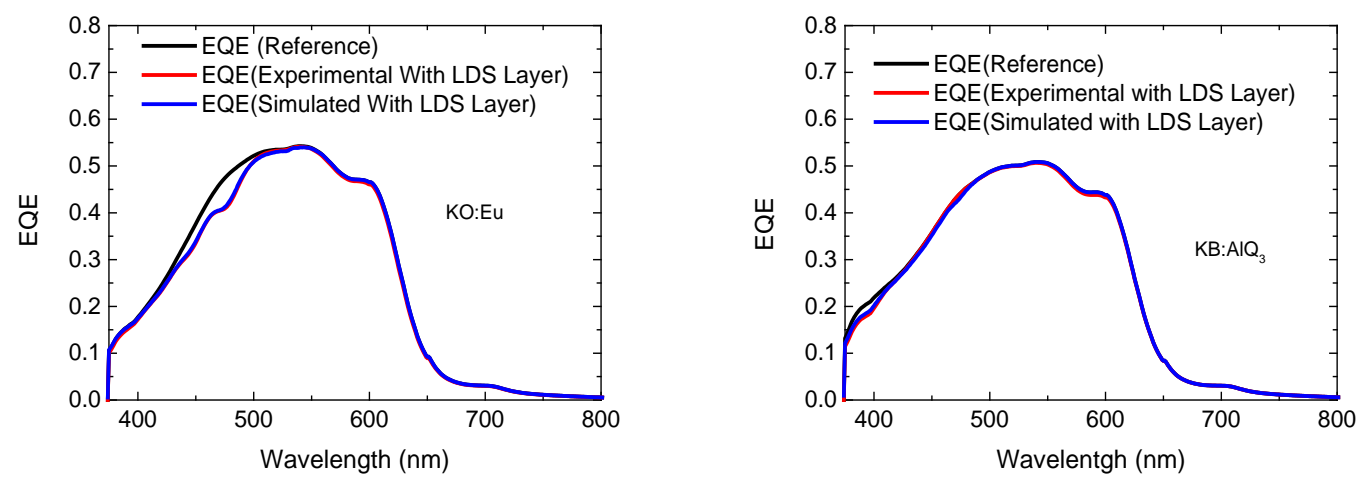

Figure S1: Experimental EQE curves for OPV without LDS layer (black line) and with LDS layer (red line), for mixtures KO:Eu (Figure $A$ ) and KB:Alq 3 (Figure B) according Fernandes et al. [32]. The simulation of the EQE curves based on the eq. (1) (see main text) is also shown in each figure (blue line).

The results of simulations are shown in Figure S1. It can be seen and accurate overlay of the experimental curve of the device with the LDS layer (red line) by the simulated curve (blue line). For the best reproduction of experimental EQE curves, the value of the nloss parameter was between 0.5 and 0.7 . Such value means that around $50-70 \%$ of the photons downshifted by the LDS layer can be used to enhance the current density generated by the photovoltaic device. 
II. Optical properties of LDS layers composed by mixtures of organic dyes.

Figure S2 contains the absorbance and PL spectra of LDS layers composed by organic dyes, both individually and in a mixture. Förster energy transfer could be possible in $\mathrm{KB}: \mathrm{C} 153$ and $\mathrm{KB}: \mathrm{C} 7$ mixtures, due to the overlap between the PL of the donor (KB) and the absorbance of the acceptor (C153 or C7). However, these curves do not overlap in the case of $\mathrm{KB}: \mathrm{Eu}$ mixture, indicating little or no Förster energy transfer are occurring.

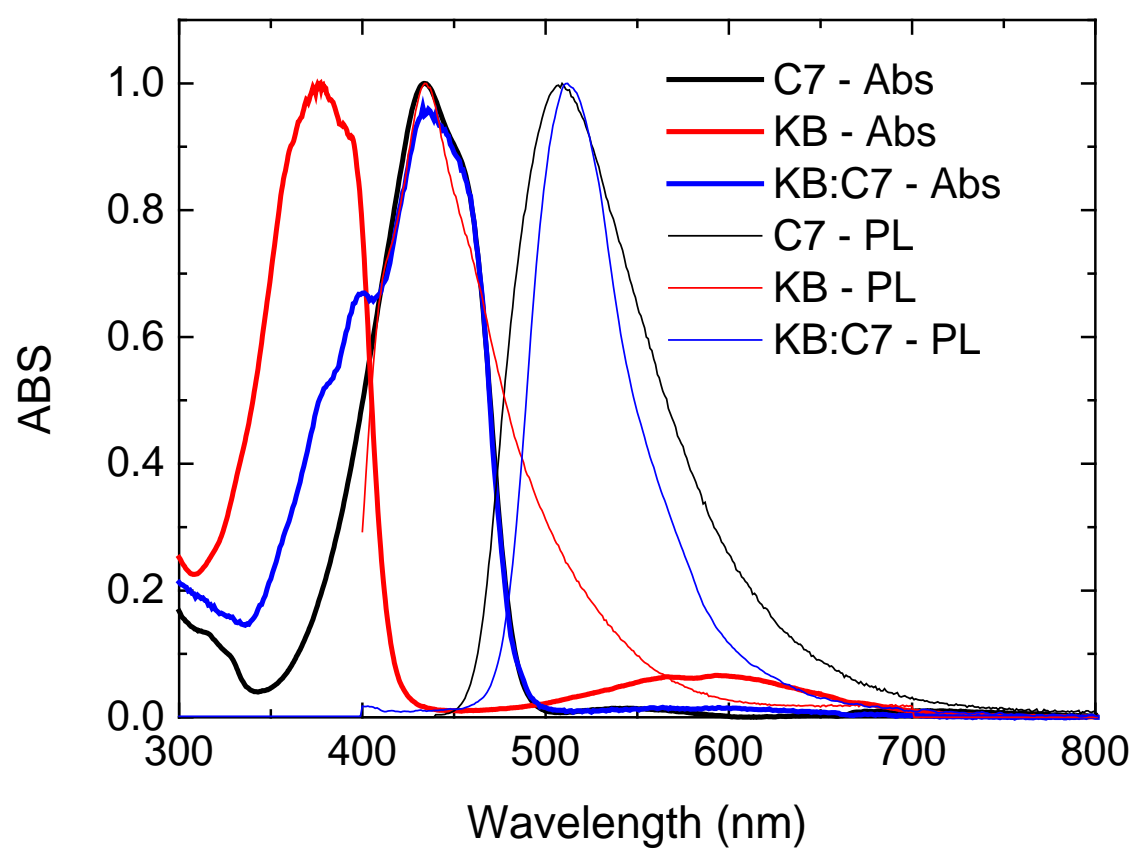



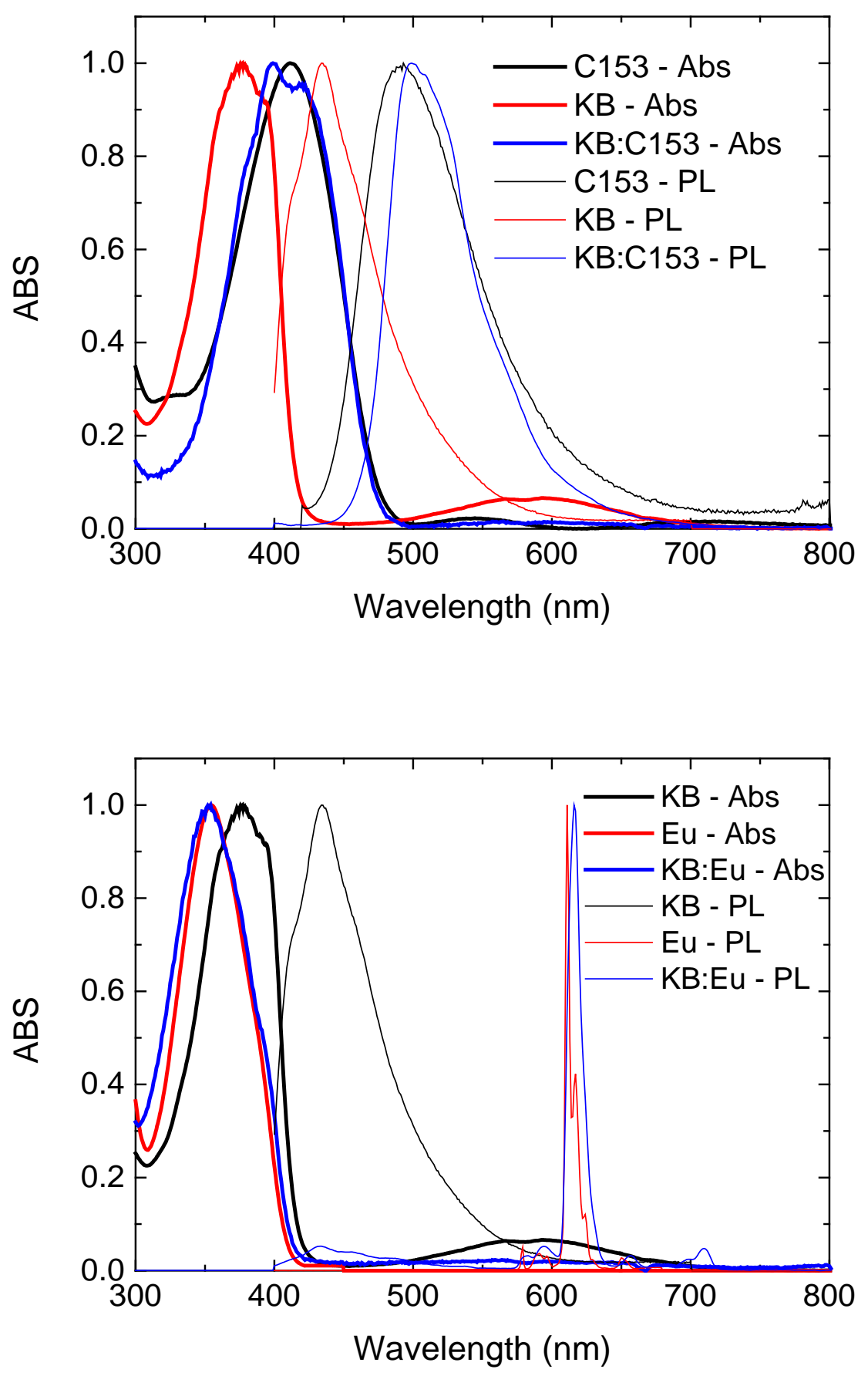

Figure S2: Absorbance and PL spectra of LDS layers composed by individuals and mixture organic dyes.

III. Tests to improve the PLQY of the LDS layers.

The first analysis was to study the influence of the following parameters on the PLQY value of the samples: (i) method for LDS layer deposition; (ii) 
amount of dripped solution; (iii) LDS material concentration in the solution; and (iv) concentration of the polymer matrix in the solution. The samples studied and their PLQY values are shown in the table SIII-1.

Table SIII-1: Samples produced from several combinations of PMMA concentration in the solution, amount of dripped solution, and concentration of luminescent material (in this case, $A / q_{3}$ ). The deposition method was tested as well. The last column shows the PLQY value of each sample listed in the table. The estimated error in the PLQY measurement was $\pm 10 \%$.

\begin{tabular}{|c|c|c|c|c|}
\hline $\begin{array}{c}\text { PMMA } \\
\text { (relative } \\
\text { weight) }\end{array}$ & $\begin{array}{c}\text { Deposition } \\
\text { method }\end{array}$ & $\begin{array}{c}\text { Amount of } \\
\text { solution } \\
(\boldsymbol{\mu} \mathrm{L})\end{array}$ & $\begin{array}{c}\text { Alq3 in } \\
\text { PMMA } \\
(\mathbf{m g} / \mathrm{mL})\end{array}$ & PLQY (\%) \\
\hline $4.00 \%$ & Drop Casting & 50 & 1.0 & 21.0 \\
\hline $4.00 \%$ & $\begin{array}{c}\text { Spin-coating } \\
(2000 \text { rpm }- \\
30 s)\end{array}$ & 50 & 1.0 & 10.2 \\
\hline $4.00 \%$ & \begin{tabular}{c} 
Drop Casting \\
\hline $4.00 \%$
\end{tabular} & $50+50$ & 1.0 & 19.1 \\
\hline $1.00 \%$ & Drop Casting & 50 & 0.1 & 16.2 \\
\hline $0.25 \%$ & Drop Casting & 50 & 1.0 & 30.0 \\
\hline
\end{tabular}

The results showed that the best values of PLQY were obtained when using drop casting method, $1 \mathrm{mg} / \mathrm{ml}$ of luminescent material, and 1\% of PMMA (in weight). The amount of material did not influence the PLQY value (but lower amount of dripped solution resulted in lower absorbance of the layer).

The same procedure was adopted for all luminescent materials selected for the work, resulting in the PLQY values reported in Table 2 of the main text.

As for the mixtures of luminescent materials, several samples were produced in accordance with the parameters listed in table SIII-2. 
Table SIII-2: Samples of LDS layers produced by mixture of the selected luminescent materials. The columns show the values for the concentration of the materials in the mixture, the amount of deposited solution, and the values of their $P L Q Y$. The estimated error in the PLQY measurement was $\pm 10 \%$.

\begin{tabular}{|c|c|c|c|}
\hline Sample & $\begin{array}{c}\text { Concentration } \\
\text { (mg:mg/mL) in } \\
\text { PMMA 1\% }\end{array}$ & Volume $(\mu \mathrm{L})$ & $\begin{array}{c}\text { PLQY } \\
\text { (\%) }\end{array}$ \\
\hline KB: C153 & $0.5: 0.5$ & 50 & 2.3 \\
\hline KB: C7 & $0.5: 0.5$ & 50 & 4.7 \\
\hline KB: Eu & $0.5: 1.0$ & 50 & 8.1 \\
\hline KB: C153 & $0.5: 0.5$ & 30 & 4.1 \\
\hline KB: C7 & $0.5: 0.5$ & 30 & 7.2 \\
\hline KB: Eu & $0.5: 1.0$ & 30 & 9.5 \\
\hline KB: C153 & $0.25: 0.25$ & 50 & 11.0 \\
\hline KB: C7 & $0.25: 0.25$ & 50 & 8.0 \\
\hline KB: Eu & $0.25: 0.5$ & 50 & 16.6 \\
\hline KB: C153 & $0.25: 0.25$ & 30 & 22.6 \\
\hline KB: C7 & $0.25: 0.25$ & 30 & 8.7 \\
\hline KB: Eu & $0.25: 0.5$ & 30 & 20.2 \\
\hline KB: C153 & $0.17: 0.17$ & 50 & 88.0 \\
\hline KB: C7 & $0.17: 0.17$ & 50 & 38.8 \\
\hline KB: Eu & $0.17: 0.33$ & 50 & 45.2 \\
\hline
\end{tabular}

It was observed that, by decreasing the concentration of the materials in the mixtures, better values were obtained for PLQY, but at the same time, the 
absorbance of the layer was lower. The conditions that led to the best compromise between absorbance and high PLQY value of the layer were used in the production of the LDS layers studied in the sequence of the work (see Table 3 of the main text). 